ates the balancing motor. This motor performs the dual purpose of balancing the radiation beams by means of a comb diaphragm placed in one of them, and of driving the pen recorder. The latter thus records the movement of the diaphragm and, therefore, the absorption of the sample placed in the other beam. The pen recorder and all the mechanical parts are of our own design.

The optical part and recording mechanism of the instrument are enclosed in a thermostatically controlled dustproof case, which can be water-cooled as well as electrically heated, and the source unit is also water-cooled. There is an inner 'Perspex' case for further protection of the prism. The whole unit is kept free from water vapour by means of drying agents, and small fans are used to circulate the air. The instrument is mounted on an antivibration assembly. The electronic amplifiers and. the power supply are mounted on a rack assembly with switching controls on the top panel.

Full details of the construction of this instrument together with performance data will be published shortly.

\section{A. BrownlIE}

W. M. CUMmrng

Technical Chemistry Department,

Royal Technical College,

Glasgow, C.1.

May 16.

${ }^{1}$ Wright, N., and Herscher, L., J. Opt. Soc. Amer., 37, 211 (1947).

' Baird, W. S., O'Bryan, H. M., Ogden, G., and Lee, D., J. Opt. Soc, Amer., 37, 754 (1947).

\section{Gauge Invariance and Rest Mass}

IT is well known that the main difference between Maxwell's equations and Proca's ${ }^{1}$ equations is that, whereas in the former the potentials $A_{\mu}$ admit the gauge transformation

$$
A_{\mu} \rightarrow A_{\mu}+\partial_{\mu} \Lambda
$$

and describe photons of zero rest mass, in the latter there is no such transformation of the potentials which describe quanta of non-zero rest mass. This is often taken to mean that in a gauge-invariant electrodynamics the rest mass of the photon must vanish. In view of the present interest in the self-energy of the photon, it seems worth while to mention that there exist gauge-invariant wave equations which can describe quanta of non-zero rest mass. For example, the equations

$$
\begin{aligned}
& F_{\mu \nu}=\partial_{\mu} A_{\nu}-\partial_{\nu} A_{\mu}, \\
& \partial^{\mu}\left(f\left(F_{\rho \sigma}, \partial_{\rho}, \partial_{\sigma} \ldots\right)+1\right) F_{\mu \nu}=j_{\nu},
\end{aligned}
$$

where $f$ is any scalar function of the field-strengths and their derivatives of any order, are consistent with the transformation (1) of potentials and with the equation $\partial^{v} j_{v}=0$, expressing the conservation of charge. It is easy to arrange for $f$ to be small for slowly varying or weak fields, so that, in these limiting cases, the equations reduce to Maxwell's. But the arbitrary function $f$ must contain some parameters which have no counterpart in Maxwell's equations, and which in certain types of theory lead to the appearance of field quanta of non-zero rest mass.

The interpretation of equation (3) is especially simple if it is linear. In this case $f$ does not involve the field-strengths and can only be a function of the d'Alembertian, $\square=\partial^{\sigma} \partial_{\sigma}$, so that (3) can be thrown into the form $\left(\lambda_{1} \square+1\right)\left(\lambda_{2} \square+1\right) \quad \ldots\left(\lambda_{n} \square+1\right) \partial \mu F_{\mu \nu}=j_{\nu}$.

Thus the field in free space satisfies

$\left(\lambda_{1} \square+1\right)\left(\lambda_{2} \square+1\right) \ldots\left(\lambda_{n} \square+1\right) \square F_{\mu \nu}=0$,

and the potentials $A_{\mu}$ satisfy a similar wave equation if they are restricted by the Lorentz condition $\partial_{\mu} A^{\mu}=0$. Equation (5) is of the general form of wave equation for a particle ${ }^{2}$ which can appear in different states of rest mass, $0, \lambda_{1}^{-1 / 2}, \lambda_{2}{ }^{-1 / 2} \ldots$. and under the influence of external forces will in general ${ }^{3}$ make transitions between these states. The generalized electrodynamics of Podolsky and Schwed falls into this general scheme.

The actual problem of the self-energy of the photon is a little different from this. One starts from the well-known set, $A$, of equations for a system of photons, electrons and heavy particles in interaction, and these equations are gauge-invariant. In the absence of free charged particles, the photons can be influenced by the virtual creation and annihilation of pairs ; but, starting from $A$, it should be possible to arrive at definite equations of motion, $B$, for photons in vacuo. All that can be said with confidence about these equations, $B$, is that they must be gauge. invariant; but as we have seen, this does not by itself imply that they cannot represent quanta with states of non-zero rest mass. The sort of complication represented by equations (3) or (5) is quite in keeping with what would be expected from the elimination of virtual-pair creation; for this elimination could scarcely be accomplished without introducing derivatives of the field-strengths.

Now suppose that one attempts to work out the energy of a photon of momentum $\hbar c \mathbf{k}$ in the absence of real charges. Since the exact solution is not known, the procedure must amount to taking the expectation value of the total Hamiltonian for some state which is only approximately a pure eigenstate and, therefore, probably has non-vanishing components in the subspaces corresponding to solutions of (3) or (5) with non-zero rest mass. In this case the expectation value of the energy will differ from $\hbar c|\mathbf{k}|$. Therefore one should not assume that gauge invariance ensures that calculations of the finite part of the energy of a photon, if pushed to high approximation, necessarily lead to the desired result $\hbar c|\mathbf{k}|$.

Actual calculations $s^{5,6,7}$ in lowest order approximation have led to various results. I wish only to remark that the finite self-energy calculated by Went$\mathrm{zel}^{7}$, being equivalent to an addition $\frac{1}{2} \gamma A_{\mu}{ }^{2}(x)$ to the photon Hamiltonian, cannot be absorbed into the theory outlined above, in which the addition to the Hamiltonian must involve derivatives of the fieldstrengths.

It is a pleasure to thank Prof. L. Rosenfeld for his interest in this work.

\section{K. J. Le Couteur}

Physical Laboratories, University, Manchester 13. May 9.

${ }^{1}$ Proca, A., J. Phys. Radium (VII), 7, 347 (1936).

2 Bhabha, H. J., Rept. Cambridge Conf., 1946, 1, 22 (1947).

a Le Couteur, K. J., Proc. Roy. Soc., A, 196, 251 (1949).

- Podolsky, B., and Schwed, P., Rev. Mod. Phys., 20, 40 (1948).

${ }^{5}$ Heisenberg, W., Z. Phys., 90, 209 (1934).

- Schwinger, J., Phys. Rev., 75, 651 (1949).

"Wentzel, G., Phys. Rev., 74, 1070 (1948). 